\title{
Effect of ionic sizes on the stability ratio of a dispersion of particles with a charge-regulated surface
}

\author{
Jyh-Ping Hsu, ${ }^{a, *}$ Shih-Wei Huang, ${ }^{a}$ and Shiojenn Tseng ${ }^{b}$ \\ a Department of Chemical Engineering, National Taiwan University, Taipei, Taiwan 10617, Republic of China \\ ${ }^{\mathrm{b}}$ Department of Mathematics, Tamkang University, Tamsui, Taiwan 25137, Republic of China
}

Received 19 June 2003; accepted 8 October 2003

\begin{abstract}
The influence of the ionic sizes on the stability of a dispersion of particles, which have an amphoteric, charged-regulated surface, is discussed. A modified Poisson-Boltzmann equation, which takes into account the sizes of ionic species, is adopted to describe the electrical field. An extended DLVO theory, which takes into account the electrical, the van der Waals, and the hydration energies, is used to estimate the stability of a colloidal dispersion. The effects of the key parameters, including ionic strength, $\mathrm{pH}$, and density of surface sites, on the behavior of problem under consideration are examined. The results obtained are qualitatively consistent with experimental findings in the literature.
\end{abstract}

(c) 2003 Elsevier Inc. All rights reserved.

Keywords: Ionic sizes; Colloidal dispersion; Stability ratio; Charge-regulated; Amphoteric surface

\section{Introduction}

The stability of a colloidal dispersion is one of its basic and significant properties. Two basic measurements are often associated with this property: the critical coagulation concentration and the stability ratio. The former is the minimum concentration of counterions necessary to initiate coagulation between particles in a stable dispersion, and the latter is the ratio (rate of fast coagulation)/(rate of slow coagulation). In conventional treatment, both of these measures are based on the classic Derjaguin-Landau-Verwey-Overbeek model (DLVO) [1], which involves two competitive interaction forces between two particles, namely, the electrostatic repulsive force and the van der Waals attractive force. Both theoretical and experimental studies relevant to the critical coagulation concentration and the stability ratio of a dispersion are ample in the literature. In particular, the advances in experimental apparatus in the past few decades facilitate more detail and accurate measurements and observations on the behavior of a dispersion. The developments in computational tools and methods also make it possible to predict the quantitative behavior of a dispersion based on a more

\footnotetext{
* Corresponding author.

E-mail address: jphsu@ccms.ntu.edu.tw (J.-P. Hsu).
}

realistic and rigorous model and to estimate its key parameters. Interactions that are not of an electrical and van der Waals nature have been proposed to elaborate experimentally observed trends that cannot be explained satisfactorily by the classic DLVO theory. These include, for example, the hydrodynamic interaction between particles [2,3]. Attempts have also been made to take into account the factors that are not considered by DLVO such as the heterogeneities on the particle surface [4-6], the charged conditions on the particle surface [7], the concentration of particles [8,9], and the finite sizes of ionic species $[10,11]$.

To evaluate the electrical interaction energy between two charged entities, the spatial variation of electrical potential needs to be known. Under certain limitations, this variation can be described by the Poisson-Boltzmann equation, which is based on Gauss's law. The analysis of Poisson-Boltzmann equation was originated by Gouy and Chapman [1], where a charged planar particle surrounded by electrolyte ions of negligible sizes in a uniform-dielectric continuum solvent was considered. Stern [12] modified their analysis through introducing the steric effects of ionic species by excluding the ions from the first molecular layer close to a charged surface. Several attempts were made to modify Stern's model by considering the effect of unequal ionic sizes on the electrical double layer [13-16], and it was shown that the sizes 
of ionic species can play a significant role in describing the properties of an electrical double layer surrounding a charged surface [17-22]. The effects of ionic sizes on the characteristics and/or phenomena of a dispersion containing particles covered by an ion-penetrable membrane, such as electrophoresis [23,24], coagulation [25], rate of flocculation [26], have also been discussed in the literature.

In a recent study, Hsu et al. [27] examined the effect of ionic sizes on the stability ratio of a colloidal dispersion for the case a particle is covered by an ion-penetrable layer, which simulates biocolloids and particles covered by an artificial membrane such as polymer. In this study, the electrical interaction between two rigid particles with a chargeregulated surface is investigated taking into account of the effect of the sizes of ionic species, and the result obtained used to evaluate the stability ratio of a colloidal dispersion based on a modified DLVO theory, which includes the hydration interaction energy between two particles. The effects of the key parameters of a dispersion, including ionic strength, $\mathrm{pH}$, and density of surface sites, on its behavior are discussed.

\section{Theory}

The analysis is begun by considering the interaction between two identical particles in an electrolyte solution. Suppose that the linear size of a particle is much larger than the thickness of the electrical double layer surrounding it, which is usually satisfied when the critical coagulation concentration is approached. Then the curvature of particle surface is insignificant, and it can be treated as a planar surface. Referring to Fig. 1, let $X, X_{\text {ca }}$, and $X_{\text {an }}$ be the scaled distance from the particle surface and the scaled effective radii of cations and of anions, respectively. Here, $X=\kappa x, X_{\mathrm{ca}}=\kappa \sigma_{\mathrm{ca}}$, and $X_{\text {an }}=\kappa \sigma_{\text {an }} ; \kappa$ and $x$ are respectively the reciprocal Debye length and the distance from the particle surface, and $\sigma_{\mathrm{ca}}$ and $\sigma_{\text {an }}$ are the effective radii of cations and of anions, re-

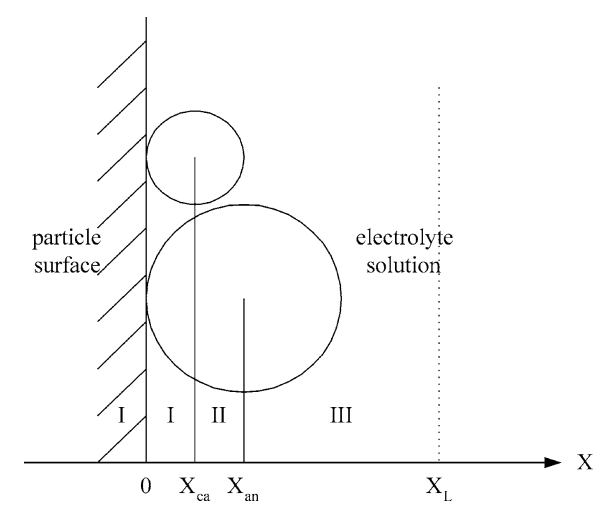

Fig. 1. Schematic representation of the problem considered. $X_{\mathrm{ca}}, X_{\mathrm{an}}$, and $X_{L}$ are respectively the scaled radius of cations, that of anions, and the scaled location of half the separation distance between two particles. Regions I-III denote respectively a charge-free region, the region in which only cations are present, and that both cations and anions are present. spectively. For illustration, we assume that $\sigma_{\text {ca }}<\sigma_{\text {an }}$; other possible cases can be treated in a similar manner. The symmetric nature of the present problem suggests that only the domain $\left(-\infty, X_{L}\right)$ needs to be considered, $X_{L}$ being the location of the middle plane between two particles. As illustrated in Fig. 1, the computational domain can be divided into three regions: region $\mathrm{I}, X<X_{\mathrm{ca}}$, which is a charge-free region; region II, $X_{\mathrm{ca}}<X<X_{\mathrm{an}}$, which contains cations only; and region III, $X_{\text {an }}<X$, which contains both cations and anions. We assume that the surface of a particle is of an amphoteric nature and is capable of undergoing the dissociation/association reactions

$\mathrm{AH} \leftrightarrow \mathrm{A}^{-}+\mathrm{H}^{+}$,

$\mathrm{AH}_{2}^{+} \leftrightarrow \mathrm{AH}+\mathrm{H}^{+}$,

where AH represents the functional group on the particle surface. The equilibrium constants associated with these reactions, $K_{-}$and $K_{+}$, can be expressed as

$K_{-}=\frac{\left[\mathrm{A}^{-}\right]\left[\mathrm{H}^{+}\right]_{s}}{[\mathrm{AH}]}$,

$K_{+}=\frac{[\mathrm{AH}]\left[\mathrm{H}^{+}\right]_{s}}{\left[\mathrm{AH}_{2}^{+}\right]}$.

Here, a symbol with a square bracket denotes a concentration, and the subscript $s$ represents a surface property. If the spatial distribution of $\mathrm{H}^{+}$follows the Boltzmann distribution, then

$\left[\mathrm{H}^{+}\right]_{s}=\left[\mathrm{H}^{+}\right]_{b} \exp (-\psi)$,

where $\left[\mathrm{H}^{+}\right]_{b}$ is the bulk concentration of $\mathrm{H}^{+}$, and $\psi$ is the scaled electrical potential, $\psi=e \phi / k_{B} T$, where $e, \phi, k_{B}$, and $T$ are respectively the elementary charge, the electrical potential, the Boltzmann constant, and the absolute temperature. If we let $C_{S}$ be the total density of available surface sites on particle surface, then

$C_{s}=\left[\mathrm{A}^{-}\right]+[\mathrm{AH}]+\left[\mathrm{AH}_{2}^{+}\right]$.

Equations (3)-(6) yield

$[\mathrm{AH}]=\frac{C_{s}}{1+K_{-} /\left[\mathrm{H}^{+}\right]_{s}+\left[\mathrm{H}^{+}\right]_{s} / K_{+}}$.

Therefore, the charge density on the particle surface, $\sigma$, can be expressed as

$\sigma=F\left(\left[\mathrm{AH}_{2}^{+}\right]-\left[\mathrm{A}^{-}\right]\right)$,

where $F$ is the Faraday constant. Equations (3), (4), (7), and (8) lead to

$\sigma=\frac{\left[\mathrm{H}^{+}\right]_{s} / K_{+}-K_{-} /\left[\mathrm{H}^{+}\right]_{s}}{1+K_{-} /\left[\mathrm{H}^{+}\right]_{s}+\left[\mathrm{H}^{+}\right]_{s} / K_{+}} C_{s}$.

The spatial variation of the electrical potential is described by the Poisson-Boltzmann equation, which is based on the Gauss law. For the present case, because the charged 
conditions in each region are different, we have

$\frac{d^{2} \psi}{d X^{2}}=0, \quad$ region I,

$\frac{d^{2} \psi}{d X^{2}}=\frac{-\exp (-a \psi)}{(a+b)}, \quad$ region II,

$\frac{d^{2} \psi}{d X^{2}}=\frac{-\exp (-a \psi)+\exp (b \psi)}{(a+b)}, \quad$ region III.

In these expressions, $\kappa^{2}=e^{2} a(a+b) n_{a}^{0} / \varepsilon_{0} \varepsilon_{r} k_{B} T$ and $b n_{b}^{0}=a n_{a}^{0}+n_{\mathrm{H}^{+}}^{0}, n_{a}^{0}$ and $n_{b}^{0}$ are respectively the bulk number concentrations of ionic species of valences $a$ and $-b$, and $n_{\mathrm{H}^{+}}^{0}$ is the bulk number concentration of $\mathrm{H}^{+}$, and $\varepsilon_{r}$ and $\varepsilon_{0}$ are the relative permittivity of the liquid phase and the permittivity of a vacuum, respectively. Suppose that both the electrical potential and the electric field are continuous at the intersection of two adjacent regions, and $\Gamma=e \sigma / \varepsilon_{0} \varepsilon_{r} k_{B} T \kappa$ denotes the scaled surface charge density. Then we have the following boundary conditions for the electric field:

$\frac{d \psi}{d X}=-\Gamma, \quad X=0$,

$\left(\frac{d \psi}{d X}\right)_{X=X_{\mathrm{ca}}^{+}}=\left(\frac{d \psi}{d X}\right)_{X=X_{\mathrm{ca}}^{-}}$and $\psi\left(X_{\mathrm{ca}}^{+}\right)=\psi\left(X_{\mathrm{ca}}^{-}\right)$,

$\left(\frac{d \psi}{d X}\right)_{X=X_{\text {an }}^{+}}=\left(\frac{d \psi}{d X}\right)_{X=X_{\text {an }}^{-}}$and $\quad \psi\left(X_{\text {an }}^{+}\right)=\psi\left(X_{\text {an }}^{-}\right)$,

$\frac{d \psi}{d X}=0, \quad X=X_{L}$.

The electrical force between two parallel, identical planar particles per unit area, $F_{R}$, can be evaluated by [27]

$\frac{F_{R}}{b n_{b}^{0} k_{B} T}=\frac{1}{a}\left[\exp \left(-a \psi_{m}\right)-1\right]+\frac{1}{b}\left[\exp \left(b \psi_{m}\right)-1\right]$,

where $\psi_{m}$ is the scaled electrical potential on the middle plane between two particles. The electrical energy between these two particles per unit area, $V_{R, p}$, can be evaluated by [22]

$V_{R, p}=\frac{2}{\kappa} \int_{X_{L}}^{\infty} F_{R} d X_{L}$.

Applying the Derjaguin approximation [1], the electrical energy between two identical spherical particles, $V_{R}$, can be estimated by

$V_{R}=\pi R_{0} \int_{X_{L}}^{\infty} V_{R, p} d X_{L}$,

where $R_{0}$ is the radius of a particle. The van der Waals energy between two identical spherical particles with a closest surface-to-surface distance $2 X_{L}, V_{A}$, is [1]

$$
\begin{aligned}
V_{A}=-\frac{A_{132}}{6}[ & \frac{R_{0}}{2 X_{L}\left(2 R_{0}+X_{L}\right)}+\frac{R_{0}^{2}}{2\left(R_{0}+X_{L}\right)^{2}} \\
& \left.+\ln \frac{X_{L}\left(2 R_{0}+X_{L}\right)}{\left(R_{0}+X_{L}\right)^{2}}\right],
\end{aligned}
$$

where $A_{132}$ is the Hamaker constant. For the case of two identical spheres with a closest surface-to-surface distance $H$, an approximate expression for the interaction energy $V_{h}$, which arises from the hydration force between them is $[28,29]$

$$
\begin{aligned}
V_{h}(H) & =\pi R_{0} \int_{H}^{\infty} P_{0} \lambda \exp \left(-\frac{H}{\lambda}\right) d H \\
& =\pi R_{0} P_{0} \lambda^{2} \exp \left(-\frac{H}{\lambda}\right),
\end{aligned}
$$

where $P_{0}$ is a hydration force constant. Experimental observation for a number of different hydrophilic solid materials reveals that $P_{0}$ ranges from $10^{6}$ to $5 \times 10^{8} \mathrm{~N} / \mathrm{m}^{2}$ [30]. $\lambda$ is a decay length constant, which is in the range from 0.2 to $1.1 \mathrm{~nm}$ for $1: 1$ electrolytes and does not vary significantly with ionic strength [31]. Molina-Bolivar et al. [32] suggested using the approximation

$V_{h}=\pi R_{0}\left(N_{A} C_{h} c_{e}\right) \lambda^{2} \exp \left(-\frac{2 X_{L}}{\lambda}\right)$,

where $C_{h}$ is a proportionality constant and $c_{e}$ is the electrolyte concentration, expressed in $\mathrm{mM}$. The total interaction energy between two particles, $V_{T}$, is the sum of the electrical, the van der Waals, and the hydration interaction energies; that is,

$V_{T}=V_{A}+V_{R}+V_{h}$.

The stability ratio for a spherical dispersion of radius $R_{0}, W$, is evaluated by [1]

$W=2 R_{0} \int_{2 R_{0}}^{\infty} \frac{1}{H^{2}} \exp \left(V_{T}\right) d H$.

\section{Results and discussion}

The influence of the key parameters of the system under consideration on its behavior is examined through numerical simulation. For illustration, both the Hamaker constant and the temperature are assumed to be constant, which implies that $V_{A}$ is a function of the separation distance between two particles only. In the numerical simulations, the ionic strength $I$, which is $I=\left(a^{2} C_{a}^{0}+b^{2} C_{b}^{0}+C_{\mathrm{H}^{+}}^{0}\right) / 2$ for the present case, varies from 1.0 to $200 \mathrm{mM}$. $C_{a}^{0}, C_{b}^{0}$, and $C_{\mathrm{H}^{+}}^{0}$ are respectively the bulk molar concentrations of cations, anions, and $\mathrm{H}^{+}$. For illustration, we assume $\mathrm{p} K_{+}=2$ and $\mathrm{p} K_{-}=9$. The isoelectric point (IEP) of an amphoteric surface can be estimated by IEP $=\left(\mathrm{p} K_{+}+\mathrm{p} K_{-}\right) / 2$ [33]. In our case, because $\mathrm{p} K_{-}=9$ and $\mathrm{p} K_{+}=2$, IEP $=5.5$. 


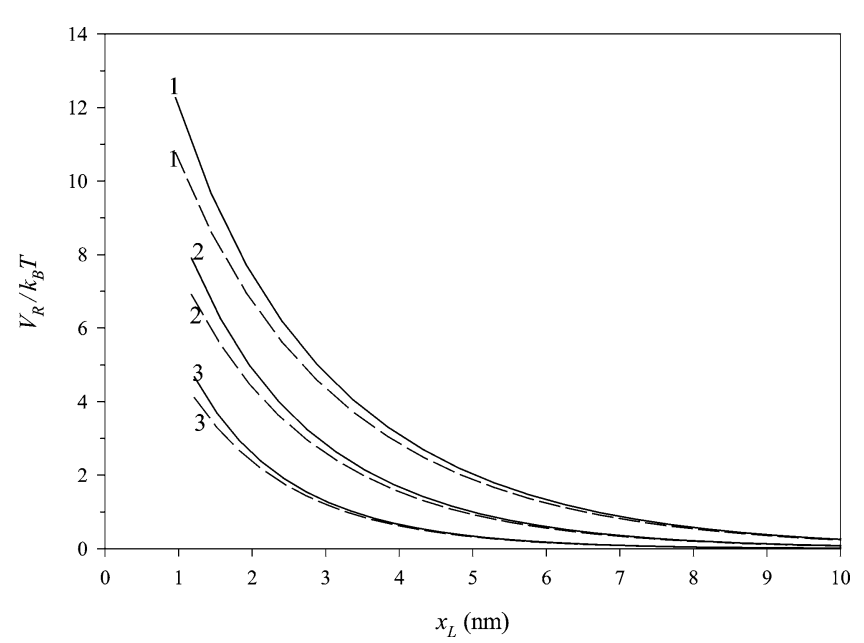

Fig. 2. Variation of scaled electrical interaction energy between two particles $V_{R} / k_{B} T$ as a function of half the separation distance between them, $x_{L}$, at various ionic strength $I$ for the case when $\sigma_{\mathrm{ca}}=0.48 \mathrm{~nm}$, $\sigma_{\mathrm{an}}=0.96 \mathrm{~nm}$, and $\mathrm{pH}$ is 7 . Solid curves, present model; dashed curves, PCM. Curve $1, I=4 \mathrm{mM}, 2, I=6 \mathrm{mM}, 3, I=10 \mathrm{mM}$. Parameters used are $T=298 \mathrm{~K}, \varepsilon_{r}=78.5, \mathrm{p} K_{-}=9, \mathrm{p} K_{+}=2, C_{s}=5 \times 10^{-6} \mathrm{~mol} / \mathrm{m}^{2}$, $a=1, b=1, X_{\mathrm{ca}}=0.05$, and $X_{\mathrm{an}}=0.1$.

Fig. 2 shows the variations in the scaled electrical interaction energy between two particles, $V_{R} / k_{B} T$, as a function of half the separation distance between them, $x_{L}$, at various ionic strengths $I$. Both the results based on the present model and those based on the corresponding point charge model (PCM) are presented. Fig. 2 indicates that, for a fixed $I$, $V_{R} / k_{B} T$ decrease with the increase in $x_{L}$, which is expected because the longer the distance between two particles the weaker the electrical interaction between them. As can be seen in Fig. 2, for a fixed $x_{L}, V_{R} / k_{B} T$ decrease with the increase in $I$. This is because an increase in $I$ has the effect of increasing the degree of screening of the surface charge by counterions. Note that according to Fig. 2, using a PCM will underestimate $V_{R} / k_{B} T$, and the lower the $I$ the more serious the deviation.

The influence of the ionic strength $I$ on the maximal scaled total interaction energy between two particles, $V_{T}^{*}$ $\left(=V_{T} / k_{B} T\right)$, is illustrated in Fig. 3. This figure shows that $V_{T}^{*}$ decreases with the increase in $I$, which implies that an increase in electrolyte concentration has the effect of increasing the probability of coagulation. Fig. 3 also indicates that the lower the ionic strength the greater the deviation in $V_{T}^{*}$ caused by neglecting the sizes of charged species. The deviation becomes insignificant, however, if $I$ is sufficiently large.

The variation of the logarithm of the stability ratio of a dispersion $W$ as a function of electrolyte concentration $C_{e}$ for the case when the $\mathrm{pH}$ of a dispersion medium is higher than the IEP is illustrated in Fig. 4. For comparison, both the result based on the present model and that based on the corresponding PCM are presented. As can be seen in Fig. 4, $W$ decreases with the increase in $C_{e}$, which is expected. This figure also reveals that PCM may underestimate appreciably

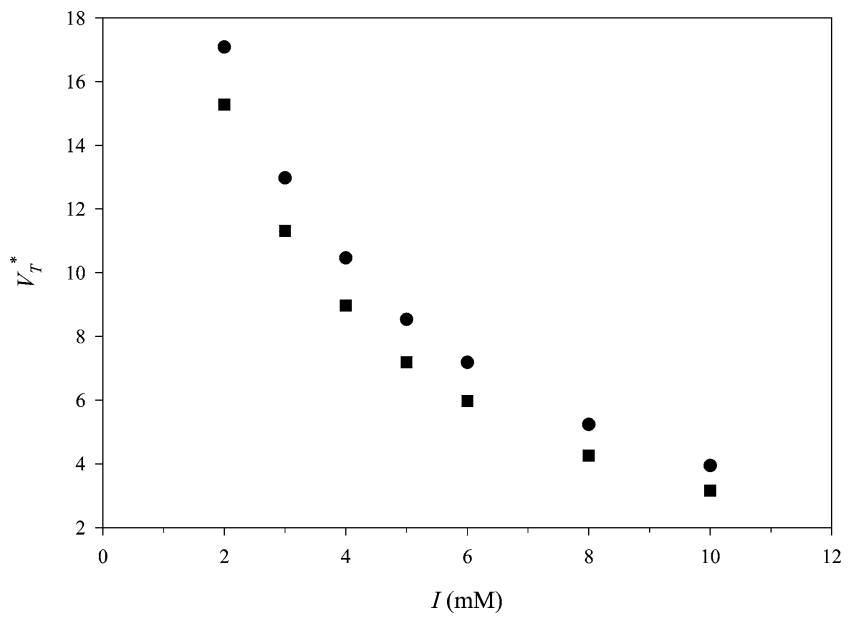

Fig. 3. Variation of maximal scaled total interaction energy between two particles, $V_{T}^{*}$, at various ionic strength $I$ for the case when $\mathrm{pH}$ is 7 , $A_{132}=2 \times 10^{-21} \mathrm{~J}, R_{0}=100 \mathrm{~nm}, \lambda=0.5 \mathrm{~nm}$, and $C_{h}=1.8 \times 10^{-20} \mathrm{~J}$. (๑) Present model, ( $\square$ ) PCM. Other parameters are the same as in Fig. 2.

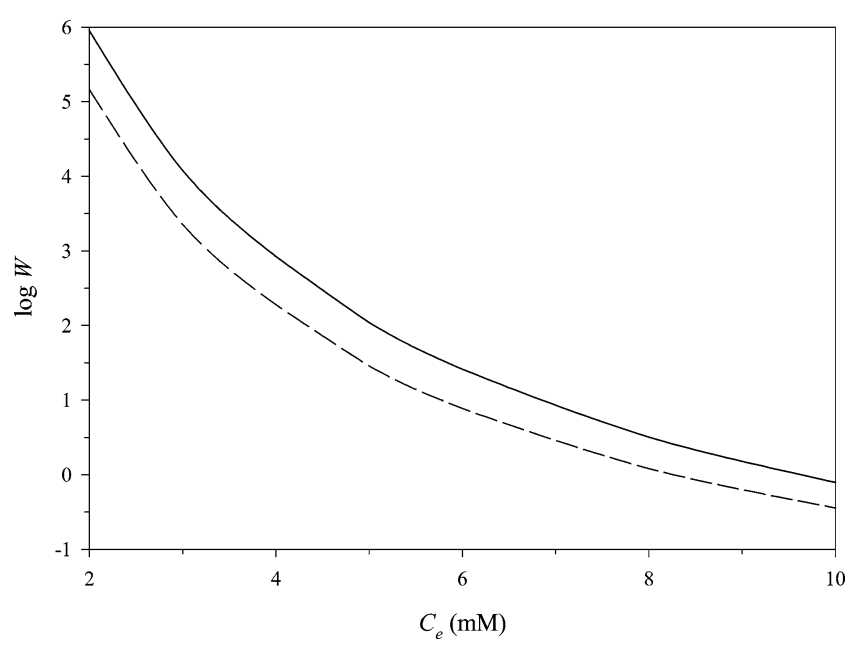

Fig. 4. Variation of the logarithm of the stability ratio of a dispersion $W$ as a function of electrolyte concentration $C_{e}$. Solid curves, present model; dashed curves, PCM. Other parameters are the same as in Fig. 3.

the stability ratio of a dispersion. For example, the deviations at $C_{e}=2,4,6$, and $10 \mathrm{mM}$ are 83.6, 77.6, 70.3, and 54.7\%, respectively. It is interesting to note that for the case where a particle is covered by an ion-penetrable membrane layer, PCM will overestimate the potential, the repulsive electrical force and energy, the total interaction energy, and the stability ratio [27], but the reverse is true if the membrane layer is absent.

The variations of the scaled electrical repulsive force between two particles, $F_{R} / b n_{b}^{0} k_{B} T$, as a function of the scaled effective radius of cations, $X_{\mathrm{ca}}$, at various $\mathrm{pH}$ 's are presented in Fig. 5. Here, the size of anions is fixed, and the $\mathrm{pH}$ of a dispersion medium is higher than the IEP; that is, particles are negatively charged. Fig. 5 indicates that for a fixed $X_{\mathrm{ca}}$, $F_{R} / b n_{b}^{0} k_{B} T$ increases with $\mathrm{pH}$. This is because if $\mathrm{pH}>$ IEP, the higher the $\mathrm{pH}$ the greater the degree of dissociation of the acidic functional groups on the particle surface, and 


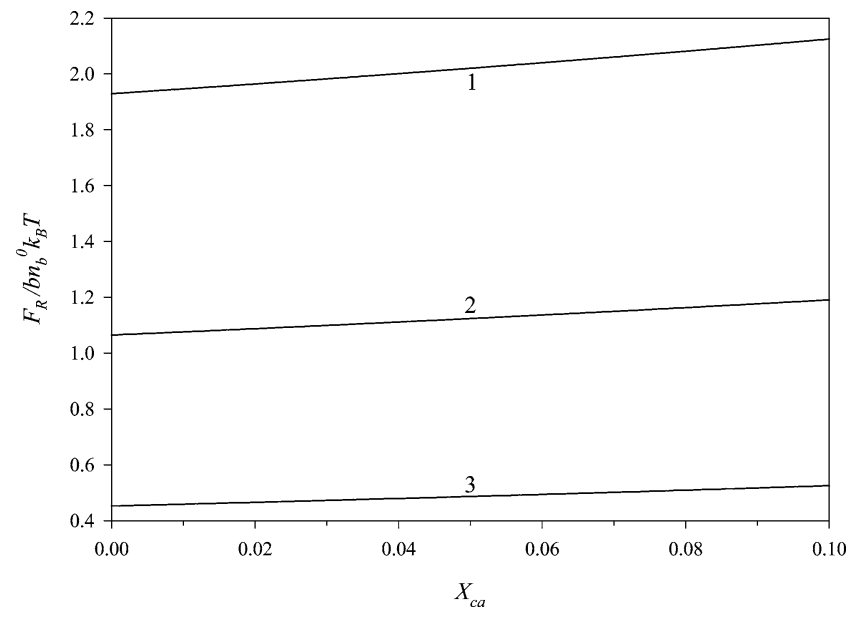

Fig. 5. Variation of scaled electrical repulsive force between two particles, $F_{R} / b n_{b}^{0} k_{B} T$, as a function of scaled effective radius of cations (counterions) $X_{\mathrm{ca}}$ at various $\mathrm{pH}$ for the case when $I=1 \mathrm{mM}, X_{\mathrm{an}}=0.1$, and

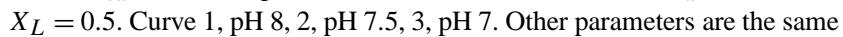
as in Fig. 2.

the higher the negative surface charge density, which leads to a greater electrical repulsive force. According to Fig. 5, $F_{R} / b n_{b}^{0} k_{B} T$ increases with the increase in $X_{\mathrm{ca}}$. In other words, the larger the effective (hydrated) size of cations (counterions) the greater the electrical repulsive force between two particles is, which is consistent with experimental observations [34]. This is because the smaller the effective size of counterions, the more closely they are able to approach the particle surface, and therefore, the more easily neutralize surface charge. Note that the corresponding PCM is unable to predict this behavior.

The influence of the scaled effective size of anions (coions), $X_{\text {an }}$, on the scaled electrical repulsive force between two particles, $F_{R} / b n_{b}^{0} k_{B} T$, for various densities of surface sites $C_{S}$ at a fixed ionic strength is illustrated in Fig. 6. Here, the size of cations is fixed, and the $\mathrm{pH}$ of a dispersion medium is higher than the IEP. Fig. 6 reveals that the higher the density of the surface sites, the larger the scaled electrical repulsive force, as expected. According to Fig. 6, the electrical repulsive force between two particles decreases with the increased size of co-ions. This is because the larger the anions (co-ions), the more difficult it is for them to get close to the particle surface, and therefore, the lower the effective negative surface charge density. A comparison between Figs. 5 and 6 indicates that the variation of the electrical repulsive force between two particles as a function of the size of co-ions is much insensitive to that as a function of the size of counterions.

The variations of the logarithm of the stability ratio of a dispersion $W$ as a function of the scaled effective radius of cations, $X_{\mathrm{ca}}\left(X_{\mathrm{an}}=0.1\right)$, and that of anions, $X_{\mathrm{an}}$ $\left(X_{\mathrm{ca}}=0.05\right)$, for the case when the $\mathrm{pH}$ of a dispersion medium is higher than IEP are presented in Fig. 7. As can be seen in this figure, the larger the $X_{\mathrm{ca}}$ or the smaller the $X_{\mathrm{an}}$, the larger the $W$. In other words, the larger the counteri-

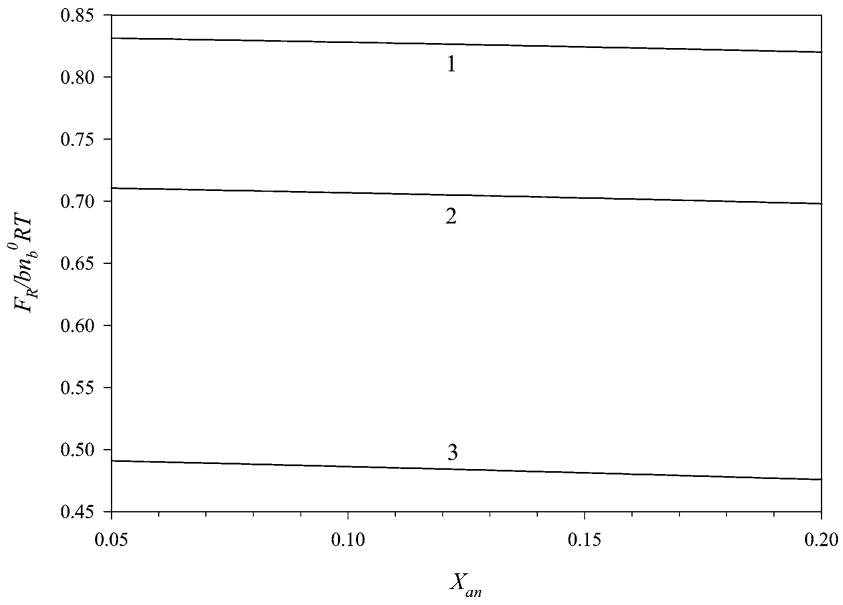

Fig. 6. Variation of scaled repulsive force between two particles, $F_{R} /$ $b n_{b}^{0} k_{B} T$, as a function of scaled effective radius of anions $X_{\text {an }}$ at various densities of surface sites, $C_{S}$, for the case when $I=1 \mathrm{mM}, X_{\mathrm{ca}}=$ $0.05, X_{L}=0.5$, and $\mathrm{pH}$ is 7 . Curve $1, C_{S}=1 \times 10^{-5} \mathrm{~mol} / \mathrm{m}^{2}, 2, C_{S}=$ $8 \times 10^{-6} \mathrm{~mol} / \mathrm{m}^{2}, 3, C_{s}=5 \times 10^{-6} \mathrm{~mol} / \mathrm{m}^{2}$. Other parameters are the same as in Fig. 2.

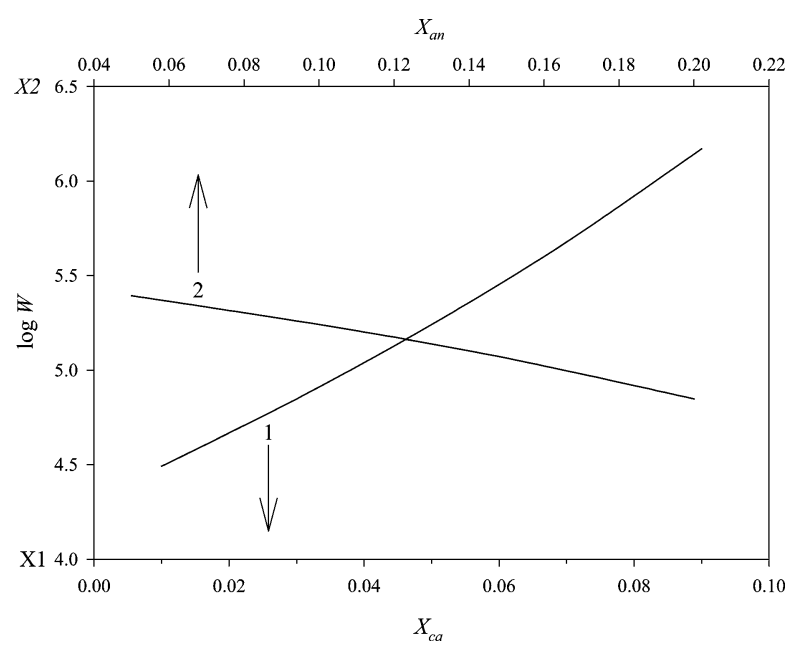

Fig. 7. Variations of the logarithm of the stability ratio of a dispersion $W$ as a function of effective sizes of cations and anions, $X_{\mathrm{ca}}$ and $X_{\mathrm{an}}$, respectively. Other parameters are the same as in Fig. 3. Curve 1 uses the $\mathrm{X} 1-\mathrm{Y}$ axis; curve 2 uses the $\mathrm{X} 2-\mathrm{Y}$ axis.

ons or the smaller the co-ions, the more stable a dispersion is. These results are consistent with experimental observations $[32,35,36]$. This figure also indicates that the variation of $W$ as a function of counterions is much sensitive to that as a function of co-ions.

\section{Conclusion}

The effects of the ionic sizes on the electrical interaction between two particles and the stability ratio of a colloidal dispersion are investigated for the case when the surface of a particle is of charge-regulated nature. We conclude that the following conditions lead to a small electrical repulsive force and a small total interaction energy between two particles: 
(i) the $\mathrm{pH}$ of dispersion medium is close to the isoelectric point of a particle, (ii) the density of available surface sites is small, (iii) the ionic strength (or concentration of electrolyte) is high, and (iv) the effective radius of counterions is small. For fixed valence of co-ions, the stability ratio of a dispersion increases with the increase in the effective radius of counterions. That is, a dispersion containing counterions of smaller effective size is less stable, which is consistent with experimental findings in the literature.

\section{Acknowledgment}

This work is supported by the National Science Council of the Republic of China.

\section{References}

[1] R.J. Hunter, Foundations of Colloid Science, vol. 1, Oxford Univ. Press, New York, 1989.

[2] L.A. Spielman, J. Colloid Interface Sci. 33 (1970) 562.

[3] M. Kostoglou, A.J. Karabelas, J. Colloid Interface Sci. 142 (1991) 297.

[4] J. Czarnecki, T. Dabros, J. Colloid Interface Sci. 78 (1980) 25.

[5] M.C. Herman, K.D. Papadopoulos, J. Colloid Interface Sci. 142 (1991) 331.

[6] D.C. Prieve, M.J. Lin, J. Colloid Interface Sci. 86 (1982) 17.

[7] J.P. Hsu, B.T. Liu, Langmuir 15 (1999) 5219.

[8] D.M. LeNeveu, R.P. Rand, V.A. Parsegian, Nature 259 (1976) 601.

[9] M.L. Broide, T.M. Tominc, M.D. Saxowsky, Phys. Rev. E 53 (1996) 6325.

[10] B. Vincent, B.H. Bijsterbosch, J. Lyklema, J. Colloid Interface Sci. 37 (1980) 171.
[11] J.L. Ortega-Vinuesa, A. Martin-Rodriguez, R. Hidalgo-Alvarez, J. Colloid Interface Sci. 184 (1996) 259.

[12] O.Z. Stern, Electrochemistry 30 (1924) 508.

[13] D. Henderson, L. Blum, J. Phys. Chem. 69 (1978) 5441.

[14] G.M. Torrie, J.P. Valleau, Chem. Phys. Lett. 65 (1979) 343.

[15] L.B. Bhuiyan, L. Blum, D. Henderson, J. Chem. Phys. 78 (1983) 442.

[16] J.P. Valleau, G.M. Torrie, J. Chem. Phys. 76 (1982) 4623.

[17] L. Blum, J. Phys. Chem. 81 (1977) 136.

[18] R. Kjellander, S. Marcelja, J. Phys. Chem. 90 (1986) 1230.

[19] R. Kjellander, T. Akesson, B. Jonsson, S. Marcelja, J. Chem. Phys. 97 (1992) 1424.

[20] J.P. Hsu, Y.C. Kuo, J. Chem. Phys. 111 (1999) 4807.

[21] Y.C. Kuo, J.P. Hsu, J. Phys. Chem. B 103 (1999) 9743.

[22] Y.C. Kuo, J.P. Hsu, Langmuir 16 (2000) 6233.

[23] S.W. Huang, J.P. Hsu, S. Tseng, Electrophoresis 22 (2001) 1881.

[24] S.W. Huang, J.P. Hsu, Y.C. Kuo, S. Tseng, J. Phys. Chem. B 106 (2002) 2117.

[25] J.P. Hsu, S.W. Huang, Y.C. Kuo, S. Tseng, J. Phys. Chem. B 106 (2002) 4269.

[26] Y.C. Kuo, J. Chem. Phys. 118 (2003) 398.

[27] J.P. Hsu, S.W. Huang, Y.C. Kuo, S. Tseng, J. Colloid Interface Sci. 262 (2003) 73.

[28] R.M. Pashley, Adv. Colloid Interface Sci. 16 (1982) 57.

[29] S. Marcelja, N. Radic, Chem. Phys. Lett. 42 (1976) 129.

[30] D.W. Thompson, I.R. Collins, J. Colloid Interface Sci. 163 (1994) 347.

[31] C.J. Van Oss, Colloids Surf. 78 (1993) 1.

[32] J.A. Molina-Bolivar, F. Galisteo-Gonzalez, R. Hidalgo-Alvarez, Colloids Surf. B 14 (1999) 3.

[33] D. Chan, T.W. Healy, L.R. White, J. Chem. Soc. Faraday Trans. 172 (1976) 2844.

[34] R.M. Pashley, J. Colloid Interface Sci. 83 (1981) 531.

[35] K.E. Verrall, P. Warwick, A.J. Fairhurst, Colloids Surf. A 150 (1999) 261.

[36] F. Dumont, P. Verbeiren, C. Buess-Herman, Colloids Surf. A 154 (1999) 149. 